\title{
Management of the axilla for ipsilateral breast cancer recurrence after previous sentinel lymph node biopsy and breast conserving therapy
}

\author{
T. Fehm • G. Helms • M. Banys • T. Kühn
}

Received: 31 May 2010 /Accepted: 1 August 2010 /Published online: 13 August 2010

(C) Springer-Verlag 2010

\begin{abstract}
The sentinel lymph node biopsy (SLNB) is the standard procedure for axillary staging in patients with primary breast cancer. Of these patients, $10-15 \%$ will develop local breast cancer recurrence. Optimal management of the axilla for these patients is still not established. The aim of this review is to assess the role of secondary lymph node biopsy in patients with ipsilateral breast cancer recurrence. The MEDLINE-PubMed databases were searched from 2006 to 2010 for publications addressing the role of secondary SLNB for ipsilateral breast cancer recurrence. Following terms were used: breast cancer recurrence, sentinel node biopsy, lymphatic drainage, follow-up lymphatic mapping. Twenty-two publications reporting a second SLNB at the time of ipsilateral relapse were identified. The detection rate of a new sentinel node ( $\mathrm{SN}$ ) was $45-97 \%$. The detection rate varied depending on the type of previous surgery. A high rate of extra-axillary re-SN was reported (32\%). In $8-35 \%$ cases, a histologically positive re-SN was diagnosed. The optimal management of the axilla in the setting of local recurrence is still not established. Prospective trials are needed to facilitate evidence-based recommendations for this situation.
\end{abstract}

Keywords Breast cancer recurrence - Sentinel node biopsy Follow-up lymphatic mapping $\cdot$ Axillary surgery

T. Fehm $(\bowtie) \cdot$ G. Helms $\cdot$ M. Banys

Universitätsfrauenklinik Tuebingen,

Calwerstr. 7 ,

72076 Tuebingen, Germany

e-mail: tanja.fehm@med.uni-tuebingen.de

M. Banys

e-mail: maggybanys@yahoo.de

T. Kühn

Frauenklinik, Klinikum Esslingen,

Hirschlandstr. 97,

73730 Esslingen, Germany

\author{
Abbreviations \\ AGO German Gynecological Oncology \\ ASCO American Society of Clinical Oncology \\ ALND axillary lymph node dissection \\ BCT breast conserving therapy \\ LSG lymphoscintigraphy \\ SLNB sentinel lymph node biopsy \\ SN sentinel node
}

\section{Background}

The objectives of primary surgical therapy of breast cancer are the removal of the tumor (therapeutic aim) and the assessment of lymph node status for selecting appropriate adjuvant treatment (diagnostic aim). Standard therapy for primary breast cancer includes breast-conserving surgery in the majority of cases. Lymph node status is determined in clinically node-negative patients by the sentinel lymph node biopsy (SLNB). The sentinel lymph node is the hypothetical first node (or several nodes) reached by cancer spread from the primary tumor $[1,2]$. This concept entails removal of selected lymph nodes at the highest risk of metastatic growth and thus the assessment of nodal status by a minimally invasive procedure. In addition, the incidence of axillary recurrence after negative findings on SLNB is comparable to that following axillary lymph node dissection (ALND) [3, 4]. ALND is indicated in the case of positive lymph nodes. The diagnostic value of ALND has drastically diminished after the introduction of SLNB and is the standard of care in cases where SLNB is not feasible [5].

Approximately $10-15 \%$ of patients after breast conserving therapy (BCT) or mastectomy suffer from local recurrence [6]. Adequate treatment for such a relapse 
following BCT consists generally of mastectomy, whereas thoracic recurrence after mastectomy is treated by wide excision [7].

The optimal surgical approach for lymph node re-staging in patients who have been previously treated with SLNB is still under debate. The exact fate of drainage patterns after previous axillary surgery or radiation therapy that alters or disrupts lymphatic pathways is not known [8,9]. Limited data from experimental studies suggest that disruption of lymphatic vessels may only be temporary and a new lymphatic network will be built up by collaterals and retained open lymphatic vessels [10]. Further research is needed to assess exact changes in lymphatic pathways and thus allow a definite statement with regard to the clinical and oncological relevance of secondary lymph node staging [11-15].

Various studies have already confirmed the feasibility and efficacy of SLNB after a biopsy of the breast [16]. Accordingly, previous breast biopsy is not considered a contraindication for SLNB in the American Society of Clinical Oncology (ASCO) recommendations [17]. However, SLNB after axillary surgery has not been widely studied. In addition, guidelines from ASCO and German Society of Senology recommend against SLNB in women who have undergone prior axillary surgery $[17,18]$. At present, there are emerging reports of successful second SLNB in patients with a local recurrence following a previous SLNB and/or axillary dissection. Therefore, the objective of this review is to discuss the potential role of second axillary sentinel node biopsy for ipsilateral breast cancer recurrence based on current literature.

\section{Methods}

The MEDLINE-PubMed databases were searched from 2006 to 2010 for publications addressing the role of secondary SLNB for ipsilateral breast cancer recurrence. The following terms were used: breast cancer recurrence, sentinel node biopsy, lymphatic drainage, and follow-up lymphatic mapping. The reference lists of selected publications were also checked for other possible relevant publications. Case reports were excluded. Twenty-two publications were identified in the PubMed database. All authors investigated the possibilities and limitations of lymph node re-staging (re-SLNB) after axillary intervention for primary breast cancer by using radionuclide labeling and lymphoscintigraphic detection of sentinel nodes.

\section{Findings}

Detection rate of SN after previous axillary surgery

The Medline research yielded 22 publications investigating the role of secondary sentinel lymph node biopsy after previous axillary surgery. The detection rate of a sentinel node (SN) after previous axillary surgery (SLNB or ALND) was $45-97 \%$ (mean $73 \%$, Table 1). In the largest study including 117 cases, a SN was identified in $55 \%$ of the patients [19]. The extent of primary axillary intervention had a significant impact on the detection of a re-SN. A new sentinel node could be identified in $78 \%$ of patients who had initially only a SLNB. In contrast, the detection rate

Table 1 Review of publications including at least 20 patients

\begin{tabular}{|c|c|c|c|c|c|c|}
\hline Author & $N$ & $\begin{array}{l}\text { Initial ALND/ } \\
\text { SLNB/none }\end{array}$ & Radiotracer injection & Detection (\%) & $\begin{array}{l}\text { SN axillary/ } \\
\text { extraaxillary }\end{array}$ & SN pos $(\%)$ \\
\hline Palit 2008 (review) [22] & 287 & $77 / 131 / 0$ & Review & 73 & n.a. $/ 32 \%$ & 15 \\
\hline Van der Ploeg 2010 [24] & 115 & $12 / 36 / 67$ & Intratumoral & 83 & $70 \% / 51 \%$ & $12 / 115(10)$ \\
\hline Port $2007^{\mathrm{a}}[19]$ & 117 & $63 / 54 / 0$ & Peritumoral $^{\mathrm{c}}$ & 55 & $69 \% / 30 \%$ & $10 / 64(16)$ \\
\hline Intra $2007^{\mathrm{a}}[20]$ & 65 & $0 / 65 / 0$ & Peritumoral & 97 & $97 \% / 8 \%$ & $7 / 65(11)$ \\
\hline Cox 2008 [38] & 56 & $0 / 56 / 0$ & Periareolar / peritumoral & 80 & $98 \% / 2 \%$ & $9 / 45(20)$ \\
\hline Axelsson 2008 [12] & 47 & $43 / 1 / 3$ & Peritumoral $^{\mathrm{d}}$ & 45 & $24 / 7$ & $7 / 20(35)$ \\
\hline Koizumi 2008 [9] & 31 & $16 / 3 / 12$ & Peritumoral & 95 & $61 \% / 36 \%$ & $4 / 28(14)$ \\
\hline Schrenk 2008 [15] & 23 & $12 / 11 / 0$ & Peritumoral & 63 & $100 \% / 31 \%$ & 3/16 (19) \\
\hline Boughey $2006^{\mathrm{a}}[8]$ & 21 & $12 / 5 / 4$ & Peritumoral & 69 & $100 \% / 46 \%$ & $1 / 13(8)$ \\
\hline Karam 2008 ${ }^{\mathrm{b}}[39]$ & 20 & $11 / 4 / 5$ & Above mastectomy scar & 75 & $15 / 2$ & $2 / 13(15)$ \\
\hline
\end{tabular}

${ }^{a}$ Included into the review by Palit et al.

${ }^{\mathrm{b}}$ Re-SLNB after previous mastectomy

${ }^{\mathrm{c}}$ Tracer injection at the axillary aspect of the recurrence site in the breast

${ }^{d}$ Tracer injection sub- or intracutaneously over the tumor 
after ALND was only $38 \%$. In addition, the identification of a re-SN was inversely correlated with the number of previously removed lymph nodes. The highest detection rate of $97 \%$ was reported by Intra et al. [20] (65 cases); however, only patients with initial breast conserving therapy and SLNB were included in the analysis.

Lymphatic drainage patterns after previous axillary surgery

Based on current reports, four main regions of lymphatic drainage in case of intramammary recurrence have been identified: ipsi- and contralateral axilla, parasternal nodes, as well as infra- and supraclavicular lymph nodes $[1,21-$ 23] (Fig. 1). Extra-axillary lymphatic drainage in case of a re-SLNB was consistently observed in up to $40 \%$ of patients (Table 1). The probability of identifying an extraaxillary SN correlated with the number of previously removed lymph nodes. The detection rate reported by Boughey et al. [8] was $50 \%$ for patients who had over ten lymph nodes removed, $30 \%$ for patients with one to ten lymph nodes and $0 \%$ for those who had no previous axillary surgery. Mastectomy also appeared to affect the rate of extra-axillary SN [8]. Van der Ploeg et al. [24] identified extra-axillary drainage in $47 \%$ of patients previously treated by BCT and SLNB. Interestingly, the percentage of extra-axillary drainage was highest $(60 \%)$ in patients who had undergone non-oncological plastic surgery. Intra et al. [20] observed no extra-axillary lympathic drainage but none of the patients had undergone a complete ALND before. In five of $63(8 \%)$ patients, an additional re-
SN outside the axilla was detected. Nonetheless, all five patients had also axillary re-SN. The type of surgery may also affect SN detection. Boughey et al. [8] reported lower $\mathrm{SN}$ identification rates in patients who had undergone previous mastectomy compared with those who had undergone breast-conserving therapy $(50 \%$ vs. $65 \%)$; however, this difference was not significant.

Positive sentinel lymph node in patients with local recurrence

The rate of metastatic re-SN in case of local relapse was observed to be between $8 \%$ and $35 \%$ (Table 1 ). The review by Palit et al. [22] reported a mean rate of $15 \%$. Interestingly, in seven out of 31 cases with extra-axillary $\mathrm{SN}$, the lymph node metastasis was localized outside the axilla. In four out of these seven patients, the metastatic node was detected in the contralateral axilla (Table 1).

\section{Discussion}

The breast develops new lymphatic drainage patterns after damage to the primary lymphatic system caused by therapy. The topography of this new pattern is significantly more variable than the one of an untreated breast. The exact mechanisms and time needed for a new drainage pattern to evolve remain to be clarified.

According to recent studies, the lymphatic pattern may depend on the type of primary surgery the patient received
Fig. 1 Frequency and localization of extra-axillary sentinel nodes (modified after Palit et al. [22]). Patients number, $n=287$; SN detection rate, $210 / 287$ (73\%); extra-axillary SN 68/210 $(32 \%)$

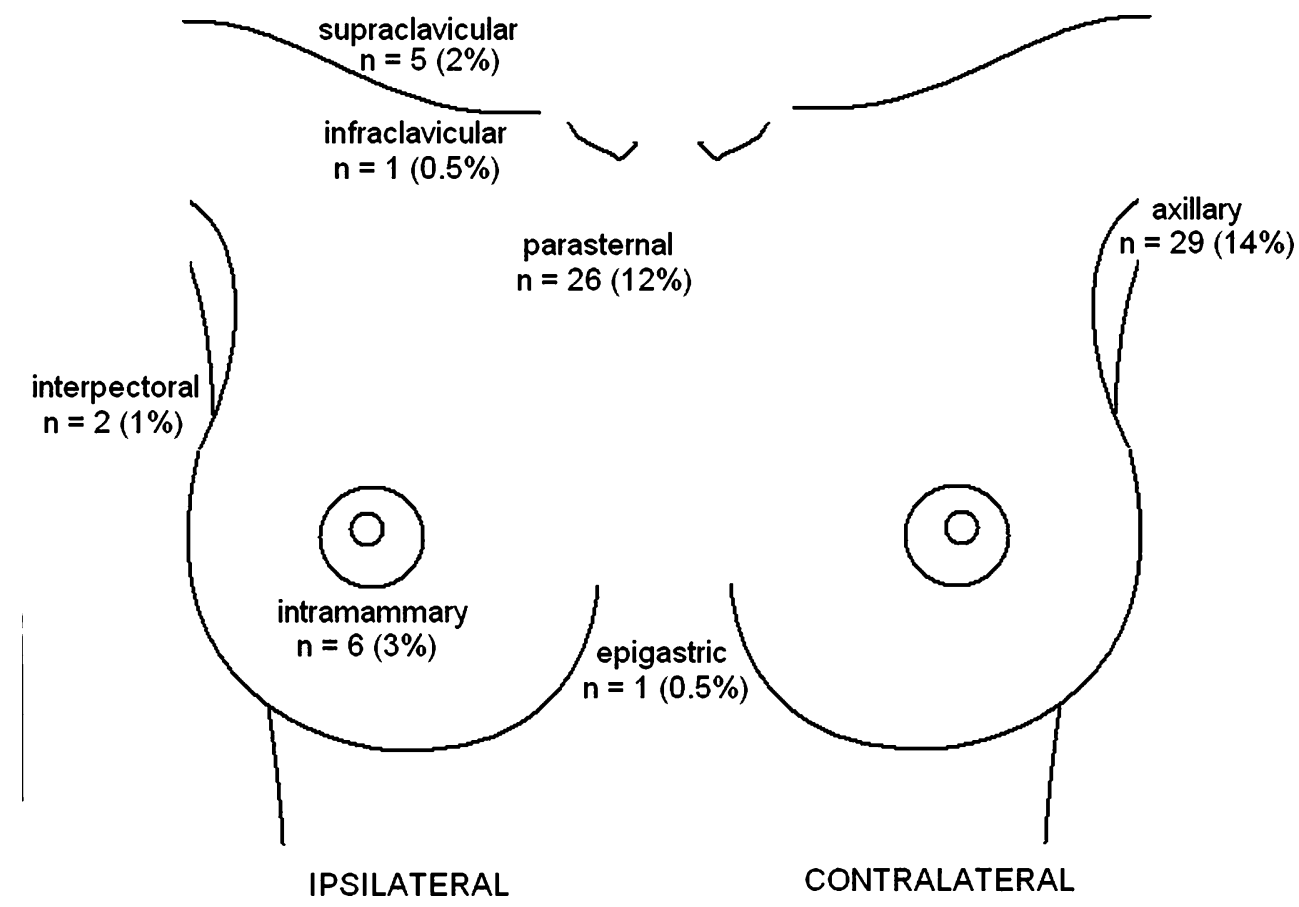


previously. The drainage system following oncological therapy (SLNB, ALND, radiation therapy) is notably variable. Consequently, mammary gland tissue and the skin do not necessarily share a common lymphatic drain (in contrast to an untreated breast). Therefore, periareolar or intradermal injection for sentinel node labeling may not match the draining pattern of the tumor. For a re-SLNB a peri- or intratumoral injection seems more appropriate. Further, lymphoscintigraphy (LSG) may contribute to the successful identification of the SN. While the clinical feasibility of LSG in primary breast cancer remains controversial, it vitally contributes to lymphatic mapping in case of local recurrence as these patients are more likely to present with an extra-axillary or contralateral sentinel node $[24,25]$.

\section{Extra-axillary sentinel nodes}

The incidence of extra-axillary drainage after prior treatment mentioned in literature is significantly higher than in non-treated breast and ranges between $20 \%$ and $67 \%$ [13, $22,24]$. This finding underlines the crucial role of lymphoscintigraphy. It is particularly important since failure to identify an extra-axillary SN may lead to down-staging of the disease and alter treatment decisions [26]. All authors (Table 1) used LSG for preoperative lymphatic mapping. In a study reported by van der Ploeg et al. [24], SPECT/CT was performed when conventional imaging failed to identify an axillary SN. The high incidence of extraaxillary drainage in a recurrent setting is a strong argument for performing a LSG [27]. Preoperative LSG reveals altered drainage patterns and allows sentinel nodes from unpredictable locations to be visualized and biopsied. Otherwise, these are sites of potential nodal involvement that would be overlooked. Thus, re-SLNB and lymphoscintigraphy may improve the surgical staging.

The clinical relevance of extra-axillary $\mathrm{SN}$ and, in particular, the internal mammary chain $\mathrm{SN}$ is under discussion [28-31]. Further, the feasibility of SLNB of the internal mammary chain nodes remains controversial as its lymphatic pattern lacks regularity observed in axillary lymph nodes. Here, the lymphatic flow from the breast reaches the internal mammary nodes through tiny vessels of each intercostal space. This raises the question of whether the 'sentinel' node concept applies to this nodal station, as there is not a node which predicts whether the other lymph nodes are involved. This observation may explain why the use of sentinel node technique in internal mammary chain does not necessarily increase nodal positivity rates $(11 \%$ in radioguided SLNB group vs. $10 \%$ in patients with biopsy of one intercostal space without sentinel technique) [26].

Development of alternative lympatic patterns depends to some extent on the type of surgery the patient received at primary operation. Boughey et al. reported increased incidence of alternative drainage in patients with $>10$ axillary lymph nodes removed previously. Similarly, successful SN detection may depend on the type of axillary surgery. Port et al. [19] from Memorial Sloan-Kettering Cancer Center reported a significantly higher SN detection rate after a previous SLNB than a previous ALND. However, current guidelines do not distinguish between previous SLNB and ALND in recommendations for therapy of local recurrence.

With regard to therapy in patients with only extra-axillary $\mathrm{SN}$, axillary lymph node dissection is typically performed. However, recent studies have shown that such patients may be spared ALND [32]. Several authors suggested that pursuing extra-axillary nodes may improve staging and lead to a better selection of patients who might benefit from radiotherapy to the internal mammary chain and from adjuvant therapy $[33,34]$. The German Gynecological Oncology (AGO) breast group currently does not make a recommendation for or against SLNB of the internal mammary chain in primary setting ( \pm , level of evidence $2 \mathrm{~b}$; Guidelines 2010, www.ago-online.de). In the case of a local ipsilateral inbreast recurrence after previous axillary surgery, AGO recommends against SLNB (level of evidence $3 b$ ).

\section{SLNB after plastic breast surgery}

Similarly, the practicability of SLNB in women with previous non-oncologic types of breast surgery such as breast augmentation or reduction mammoplasty remains unclear. Guidelines from ASCO and AGO did not make a recommendation for or against SLNB in these cases due to insufficient data (AGO guideline: level of evidence 4). However, recent studies confirm the feasibility of the SLNB technique for identifying the sentinel node in patients with previous esthetic breast surgery. Rodriguez Fernandez et al. reported a SN identification rate of $100 \%$ in patients with previous esthetic surgery (augmentation or reduction). All patients underwent lymphoscintigraphy prior to surgery [25]. Several smaller studies suggest that SLNB may also be feasible after transaxillary approach $[35,36]$. Interestingly, van der Ploeg et al. [24] describe a high percentage $(60 \%)$ of extra-axillary drainage in patients who had undergone plastic surgery.

\section{SLNB after radiation therapy}

Radiation therapy of the breast or its regional lymph nodes may alter lymphatic patterns. This may have consequences for lymphatic mapping and SN detection afterwards. Recently, van der Ploeg et al. [37] examined lymphatic drainage in 22 breast cancer patients who underwent mantle field radiation therapy for Hodgkin's lymphoma in the past. 
This treatment includes radiation of axillary, supra- and infraclavicular, mediastinal lymph nodes. Lymphatic y4mapping yielded a lymph node in $86 \%$ of the patients. Nonvisualization and extra-axillary SN were more frequently encountered than in patients without history of radiation therapy. Similarly, the detection of contralateral sentinel nodes in patients with breast cancer recurrence seems to increase after previous radiation therapy. Koizumi et al. [9] showed lymphatic drainage to contralateral nodes in seven out of ten cases with previous irradiation.

Clinical relevance of lymph node status in recurrent breast cancer

Another important issue is the purpose of SLNB in relapse setting. Up to $35 \%$ of patients who are diagnosed with local recurrence have histologically positive lymph nodes. Whether the removal of metastatic nodes may improve local control of the disease, and thus disease-free survival, is currently being debated. Current guidelines do not provide universal recommendations for or against axillary surgery in recurrent cases. Regarding diagnostic value, positive node status may imply more aggressive or advanced disease. Thus, nodal assessment may contribute to optimized staging and improve treatment decisions. Interestingly, a high rate of extra-axillary and even contralateral SN has been observed. The question remains as to whether contralateral re-SN metastasis should be classified as an M1 (palliative) situation or a locally restricted and thus curable disease. Contralateral metastatic lymph nodes are mostly diagnosed after previous axillary dissection [24]. In such cases, contralateral nodes may merely reflect the nearest direct drainage site and not distant metastasis.

What is the clinical and prognostic relevance of positive re-SN status in comparison to primary disease? No consensus has been reached so far regarding the optimal systemic therapy for breast cancer local recurrence. Future studies must clarify whether selected patients' groups benefit from secondary chemotherapy. Reevaluation of lymph node status may provide additional data and thus enable individualized and optimized treatment.

\section{Conclusions}

The adequate treatment of lymphatic pathways in the case of ipsilateral breast cancer recurrence remains to be clarified. In primary breast cancer, SLNB is a staging procedure that provides prognostic information for tailored adjuvant treatment. In contrast, the clinical implications of a positive lymph node in the case of a local recurrence are not defined. Chemotherapy is in general not recommended in this situation. The oncologic significance of positive nodes with respect to prognosis and further tumor spread is unclear. Whether patients with a positive re-SN derive any benefit from adjuvant chemotherapy or radiotherapy is highly speculative and deserves further evaluation.

Current data is insufficient due to small patient groups, inhomogeneous collectives and partly contradictory results. However, review of the literature provides important insights into the development of new lymphatic pathways of the treated breast. Since we will be confronted more often with local recurrences in patients treated only with SLNB, an evidence-based recommendation for re-SLNB is necessary.

Although the role of re-SLNB remains unclear, the following clinical recommendations can be derived from this review: a re-SLNB is feasible in the majority of patients who have undergone previous SLNB. Re-SLNB can be performed in order to detect and remove tumor burden in the lymph nodes. The procedure does, however, not appear mandatory. Axillary dissection is not indicated if an SN cannot be detected.

Acknowledgments We thank Anna Sophia McKenney (Cornell University, NY) for revising the manuscript.

Declaration of interest The authors report no conflicts of interest. The authors alone are responsible for the content and writing of the paper.

\section{References}

1. Sood A, Youssef IM, Heiba SI, El-Zeftawy H, Axelrod D, Seigel B et al (2004) Alternative lymphatic pathway after previous axillary node dissection in recurrent/primary breast cancer. Clin Nucl Med 29:698 702

2. Luini A, Galimberti V, Gatti G, Arnone P, Vento AR, Trifiro $G$ et al (2005) The sentinel node biopsy after previous breast surgery: preliminary results on 543 patients treated at the European Institute of Oncology. Breast Cancer Res Treat 89: 159-163

3. Veronesi U, Paganelli G, Viale G, Luini A, Zurrida S, Galimberti Vet al (2003) A randomized comparison of sentinel-node biopsy with routine axillary dissection in breast cancer. N Engl J Med 349:546553

4. Naik AM, Fey J, Gemignani M, Heerdt A, Montgomery L, Petrek $\mathrm{J}$ et al (2004) The risk of axillary relapse after sentinel lymph node biopsy for breast cancer is comparable with that of axillary lymph node dissection: a follow-up study of 4,008 procedures. Ann Surg 240:462-468, discussion 68-71

5. Takei H, Kurosumi M, Yoshida T, Ninomiya J, Hagiwara Y, Kamimura $\mathrm{M}$ et al (2007) Current trends of sentinel lymph node biopsy for breast cancer - a surgeon's perspective. Breast Cancer 14:362-370

6. Silva E, Zurrida S (2005) Breast cancer, a practical guide. Elsevier, Saunders

7. Wockel A, Kreienberg R (2008) First revision of the German S3 guideline 'diagnosis, therapy, and follow-up of breast cancer'. Breast Care 3:82-86 
8. Boughey JC, Ross MI, Babiera GV, Bedrosian I, Feig BW, Hwang RF et al (2006) Sentinel lymph node surgery in locally recurrent breast cancer. Clin Breast Cancer 7:248-253

9. Koizumi M, Koyama M, Tada K, Nishimura S, Miyagi Y, Makita $M$ et al (2008) The feasibility of sentinel node biopsy in the previously treated breast. Eur J Surg Oncol 34:365-368

10. Halsell JT, Smith JR, Bentlage CR, Park OK, Humphreys JW Jr (1965) Lymphatic drainage of the breast demonstrated by vital dye staining and radiography. Ann Surg 162:221-226

11. Agarwal A, Heron DE, Sumkin J, Falk J (2005) Contralateral uptake and metastases in sentinel lymph node mapping for recurrent breast cancer. J Surg Oncol 92:4-8

12. Axelsson CK, Jonsson PE (2008) Sentinel lymph node biopsy in operations for recurrent breast cancer. Eur J Surg Oncol 34:626-630

13. Newman EA, Cimmino VM, Sabel MS, Diehl KM, Frey KA, Chang AE et al (2006) Lymphatic mapping and sentinel lymph node biopsy for patients with local recurrence after breastconservation therapy. Ann Surg Oncol 13:52-57

14. Roumen RM, Kuijt GP, Liem IH (2006) Lymphatic mapping and sentinel node harvesting in patients with recurrent breast cancer. Eur J Surg Oncol 32:1076-1081

15. Schrenk P, Tausch C, Wayand W (2008) Lymphatic mapping in patients with primary or recurrent breast cancer following previous axillary surgery. Eur J Surg Oncol 34:851-856

16. Heuts EM, van der Ent FW, Kengen RA, van der Pol HA, Hulsewe KW, Hoofwijk AG (2006) Results of sentinel node biopsy not affected by previous excisional biopsy. Eur J Surg Oncol 32:278-281

17. Lyman GH, Giuliano AE, Somerfield MR, Benson AB 3rd, Bodurka DC, Burstein HJ et al (2005) American society of clinical oncology guideline recommendations for sentinel lymph node biopsy in early-stage breast cancer. J Clin Oncol 23:77037720

18. Kuehn T, Bembenek A, Decker T, Munz DL, Sautter-Bihl ML, Untch $\mathrm{M}$ et al (2005) A concept for the clinical implementation of sentinel lymph node biopsy in patients with breast carcinoma with special regard to quality assurance. Cancer 103:451-461

19. Port ER, Garcia-Etienne CA, Park J, Fey J, Borgen PI, Cody HS 3rd (2007) Reoperative sentinel lymph node biopsy: a new frontier in the management of ipsilateral breast tumor recurrence. Ann Surg Oncol 14:2209-2214

20. Intra $M$, Trifiro $G$, Galimberti V, Gentilini O, Rotmensz N, Veronesi P (2007) Second axillary sentinel node biopsy for ipsilateral breast tumour recurrence. Br J Surg 94:1216-1219

21. Dinan D, Nagle CE, Pettinga J (2005) Lymphatic mapping and sentinel node biopsy in women with an ipsilateral second breast carcinoma and a history of breast and axillary surgery. Am J Surg 190:614-617

22. Palit G, Jacquemyn Y, Tjalma W (2008) Sentinel node biopsy for ipsilateral breast cancer recurrence: a review. Eur J Gynaecol Oncol 29:565-567

23. Taback B, Nguyen P, Hansen N, Edwards GK, Conway K, Giuliano AE (2006) Sentinel lymph node biopsy for local recurrence of breast cancer after breast-conserving therapy. Ann Surg Oncol 13:1099-1104
24. van der Ploeg IM, Oldenburg HS, Rutgers EJ, Baas-Vrancken Peeters MJ, Kroon BB, Valdes Olmos RA et al (2010) Lymphatic drainage patterns from the treated breast. Ann Surg Oncol 17:1069-1075

25. Rodriguez Fernandez J, Martella S, Trifiro G, Caliskan M, Chifu C, Brenelli F et al (2009) Sentinel node biopsy in patients with previous breast aesthetic surgery. Ann Surg Oncol 16:989-992

26. Veronesi U, Arnone P, Veronesi P, Galimberti V, Luini A, Rotmensz N et al (2008) The value of radiotherapy on metastatic internal mammary nodes in breast cancer. Results on a large series. Ann Oncol 19:1553-1560

27. Tasevski R, Gogos AJ, Mann GB (2009) Reoperative sentinel lymph node biopsy in ipsilateral breast cancer relapse. Breast 18:322-326

28. Sugg SL, Ferguson DJ, Posner MC, Heimann R (2000) Should internal mammary nodes be sampled in the sentinel lymph node era? Ann Surg Oncol 7:188-192

29. Bourre JC, Payan R, Collomb D, Gallazzini-Crepin C, Calizzano A, Desruet MD et al (2009) Can the sentinel lymph node technique affect decisions to offer internal mammary chain irradiation? Eur J Nucl Med Mol Imaging 36:758-764

30. Yao MS, Kurland BF, Smith AH, Schubert EK, Dunnwald LK, Byrd DR et al (2007) Internal mammary nodal chain drainage is a prognostic indicator in axillary node-positive breast cancer. Ann Surg Oncol 14:2985-2993

31. Cody HS 3rd (2010) Clinical significance and management of extra-axillary sentinel lymph nodes: worthwhile or irrelevant? Surg Oncol Clin N Am 19:507-517

32. van der Ploeg IM, Tanis PJ, Valdes Olmos RA, Kroon BB, Rutgers EJ, Nieweg OE (2008) Breast cancer patients with extraaxillary sentinel nodes only may be spared axillary lymph node dissection. Ann Surg Oncol 15:3239-3243

33. Estourgie SH, Nieweg OE, Olmos RA, Rutgers EJ, Kroon BB (2004) Lymphatic drainage patterns from the breast. Ann Surg 239:232-237

34. Chen RC, Lin NU, Golshan M, Harris JR, Bellon JR (2008) Internal mammary nodes in breast cancer: diagnosis and implications for patient management - a systematic review. J Clin Oncol 26:4981-4989

35. Munhoz AM, Aldrighi C, Ono C, Buchpiguel C, Montag E, Fells $\mathrm{K}$ et al (2007) The influence of subfascial transaxillary breast augmentation in axillary lymphatic drainage patterns and sentinel lymph node detection. Ann Plast Surg 58:141-149

36. Mottura AA, Del Castillo R (2007) Transaxillary breast augmentation: two breast cancer patients with successful sentinel lymph node diagnosis. Aesthet Plast Surg 31:544-549, discussion 50-2

37. van der Ploeg IM, Russell NS, Nieweg OE, Oldenburg HS, Kroon $\mathrm{BB}$, Olmos RA et al (2009) Lymphatic drainage patterns in breast cancer patients who previously underwent mantle field radiation. Ann Surg Oncol 16:2295-2299

38. Cox CE, Furman BT, Kiluk JV, Jara J, Koeppel W, Meade T et al (2008) Use of reoperative sentinel lymph node biopsy in breast cancer patients. J Am Coll Surg 207:57-61

39. Karam A, Stempel M, Cody HS 3rd, Port ER (2008) Reoperative sentinel lymph node biopsy after previous mastectomy. J Am Coll Surg 207:543-548 